\title{
Role of spin-orbit coupling in the far infrared absorption of lateral semiconductor dots
}

\author{
Manuel Valín-Rodríguez, Antonio Puente, and Llorenç Serra \\ Departament de Física, Universitat de les Illes Balears, E-07071 Palma de Mallorca, Spain
}

(December 10, 2001)

\begin{abstract}
We investigate the relevance of the spin-orbit coupling to the far-infrared absorption of two-dimensional semiconductor dots. Varying the strength of the Dresselhaus term, a mechanism feasible in experiment by changing the dot width, distinctive splittings of the Kohn peak as well as additional low energy modes are predicted in a non-interacting model. Each mode has a spatial distribution of charge, perpendicular and in-plane spin densities that correlate in a peculiar way with the frequency and polarization of the external field. We study the robustness of these features against electron-electron interactions as well as the appearance of interaction-induced additional characteristics.
\end{abstract}

PACS 73.21.La, 73.21.-b

The role of the electron spin-orbit (SO) coupling in the general properties of lateral semiconductor dots has attracted much interest in recent years. To a large extent this is motivated by the technological impact attributed to the so-called spintronic devices, whose operation principle exploits precisely the SO mechanism to control the spin transport [1]. Actually, spintronics is an incipient field whose fundamental principles need a deeper understanding [2]. In this general context, experimental and theoretical investigations have very recently shown that the SO coupling affects the quantum transport and, more specifically, the conductance fluctuations of chaotic quantum dots in a parallel magnetic field [3 5].

The different SO contributions in semiconductor dots have been reviewed by Voskoboynikov et al. [6]. In general, SO coupling has two contributions: the Rashba term due to the nanostructure confinement potential; and the Dresselhaus term originating from the crystalline inversion asymmetry. In lateral dots the strength of the latter depends on the vertical width, which can be experimentally varied with vertical electric fields. For an embedding GaAs medium both Rashba and Dresselhaus parameters are reasonably determined [7].

In this Letter we study the influence of SO couplings on the dipole absorption of GaAs dots with a parabolic confinement. While the Rashba term provides a negligible effect we show that the Dresselhaus contribution leads to characteristic splittings of the Kohn modes and to the appearance of quasi-spin flip modes in the far-infrared absorption (FIR). For each mode we find a correlation between the spatial patterns of oscillating electron density, horizontal (in-plane) spin density, and vertical spin density. As in most of the literature treating the SO coupling [4 [6], we shall first consider a non-interacting model, using both analytical and numerical treatments. Nevertheless, the role of the interaction will be also considered within self-consistent mean-field for Hartree and exchange-correlation contributions, using a spinorial formalism of local-spin-density theory (LSDA) [8].

Within the effective Hamiltonian formalism and for a quasi two-dimensional system the Rashba and Dresselhaus [for the standard (001) plane of GaAs] contributions read, respectively,

$$
\begin{aligned}
& \mathcal{H}_{R}=\frac{\lambda_{R}}{\hbar} \sum_{i=1}^{N}[\vec{\sigma} \cdot(\nabla V(\mathbf{r}) \times \mathbf{P})]_{i} \\
& \mathcal{H}_{D}=\frac{\lambda_{D}}{\hbar} \sum_{i=1}^{N}\left[P_{x} \sigma_{x}-P_{y} \sigma_{y}\right]_{i},
\end{aligned}
$$

where the $\lambda$ 's control the coupling strengths, the $\sigma$ 's refer to the Pauli matrices and $V(\mathbf{r})$ stands for the total electrostatic potential. In Eq. (1), $\mathbf{P}$ gives the kinetic momentum. Although we shall not consider in this work an external magnetic field (or vector potential A) it can be easily included using the canonical momentum $\mathbf{P}=-i \hbar \nabla+\frac{e}{c} \mathbf{A}$ in Eq. (1).

For GaAs one has $\lambda_{R}=5.33 \AA^{2}$ and a material-specific constant $\gamma=27.5 \mathrm{eV}^{3}$ [7]. The latter determines the Dresselhaus coefficient in terms of the dot vertical width $z_{0}$ as [6] $\lambda_{D} \approx \gamma\left(\pi / z_{0}\right)^{2}$. As a representative case, we consider an external parabolic confinement with energy $\hbar \omega_{0}=4.2 \mathrm{meV}$ and a number of electrons ranging from 6 to 12 . A strict two-dimensional motion is assumed and the dot width is simply taken as a parameter for the Dresselhaus coupling. The Rashba term turns out to be negligible in all cases we have studied. However, the Dresselhaus term increases its importance for decreasing doth widths, leading to relevant effects as we shall show below already for widths $z_{0} \approx 50 \AA$. The influence of the Rashba term has already been studied in the literature for InSb dots [9], where band non-parabolicity is important, and in phenomenologically fitted models [10].

We have implemented the numerical solution on a spatial grid of the full Hamiltonian $\mathcal{H}_{\text {full }}=\mathcal{H}_{0}+\mathcal{H}_{R}+\mathcal{H}_{D}$, with

$$
\mathcal{H}_{0}=\sum_{i=1}^{N}\left[\frac{\mathbf{P}^{2}}{2 m}+\frac{1}{2} m \omega_{0}^{2} r^{2}\right]_{i} .
$$

However, before presenting numerical results it is worth to obtain an approximate analytical solution of the relevant contributions $\mathcal{H}_{0}+\mathcal{H}_{D} \equiv \mathcal{H}$ with an approach 
similar to that of Aleiner and Fal'ko [5]. A diagonalization to second order in $\lambda_{D}$ is obtained by means of the transformation $\tilde{\mathcal{H}}=U^{+} \mathcal{H} U$, where

$$
U=\exp \left[-i \lambda_{D} \frac{m}{\hbar^{2}} \sum_{j=1}^{N}\left(x \sigma_{x}-y \sigma_{y}\right)_{j}\right] .
$$

We find

$$
\begin{aligned}
\tilde{\mathcal{H}} & =\sum_{j=1}^{N}\left[\frac{\mathbf{P}^{2}}{2 m}+\frac{1}{2} m \omega_{0}^{2} r^{2}+\lambda_{D}^{2} \frac{m}{\hbar^{3}}\left(x P_{y}-y P_{x}\right) \sigma_{z}\right]_{j} \\
& -N \lambda_{D}^{2} \frac{m}{\hbar^{2}}+O\left(\lambda_{D}^{3}\right) .
\end{aligned}
$$

Identifying the orbital angular momentum $\ell_{z}=x P_{y}-$ $y P_{x}$ in (身), we realize that the Dresselhaus term introduces a correction depending on $\ell_{z} \sigma_{z}$ quite similar to that of the Rashba term for a circular system, but in the transformed reference frame. In fact, in the laboratory frame the $\mathcal{H}$ eigenstates to first order in $\lambda_{D}$ are spinors that slightly deviate from the $\sigma_{z}$ eigenspinors. We shall call them quasi-up $\chi_{q u}$ and quasi-down $\chi_{q d}$ states

$$
\begin{aligned}
\chi_{q u} & \equiv \varphi_{n \ell}(\mathbf{r})\left(\begin{array}{c}
1 \\
-\lambda_{D} \frac{m}{\hbar^{2}}(y+i x)
\end{array}\right), \\
\chi_{q d} & \equiv \varphi_{n \ell}(\mathbf{r})\left(\begin{array}{c}
\lambda_{D} \frac{m}{\hbar^{2}}(y-i x) \\
1
\end{array}\right),
\end{aligned}
$$

where $\varphi_{n \ell}(\mathbf{r})$ are the eigenfunctions of the circular harmonic oscillator $\mathcal{H}_{0}$. Since the dipole operator is invariant by the transformation (3), the analytical solution predicts dipole transitions with $\Delta \ell= \pm 1$ for quasi-up and quasi-down subsystems (see Fig. 1) at the energies

$$
\begin{aligned}
& \hbar \omega_{q u, \pm 1}=\hbar \omega_{0} \pm \lambda_{D}^{2} \frac{m}{\hbar^{2}} \\
& \hbar \omega_{q d, \pm 1}=\hbar \omega_{0} \mp \lambda_{D}^{2} \frac{m}{\hbar^{2}} .
\end{aligned}
$$

An inmediate consequence is the splitting of the original Kohn mode at $\hbar \omega_{0}$ in two peaks separated by an energy $\Delta \hbar \omega=2 \lambda_{D}^{2} m / \hbar^{2}$.

Left panels of Fig. 2 show the numerical result for the FIR absorption of $\mathcal{H}_{\text {full }}$ obtained within a real-time simulation after an initial rigid displacement of the system as in Ref. [11]. The results correspond to $N=6$ and 10 electrons with a Dresselhaus parameter for a dot width of $z_{0}=50 \AA$. The splitting around $\hbar \omega_{0}$ nicely agrees with the analytical prediction (arrows), thus proving the validity of the model. For the $N=10$ case we note the existence at low energy of a peak with much less strength than the dominant ones (notice the enlarged scale). This too can be explained within the analytical model realizing that $N=10$ has a partial occupation of the third oscillator major shell and thus the possibility of quasispin flip transitions $\chi_{q u} \leftrightarrow \chi_{q d}$ at an energy $4 \lambda_{D}^{2} m / \hbar^{2}$, indicated with dotted lines in Fig. 1 and with an arrow in Fig. 2. Note that in spite of having $\Delta \ell_{z}=0$ these transitions can manifest in the dipole spectrum through the higher order corrections in Eq. (何).

Due to the spin-orbit coupling the charge dipole oscillation also induces a spin oscillation. This is proved in the right panels of Fig. 2 where the Fourier transform of $\left\langle S_{x}+S_{y}\right\rangle$ is plotted. The same frequencies of the dipole absorption manifest in the spin channels, although with different relative strengths. It is also interesting to analyze the spatial patterns of induced density $\delta \rho(\mathbf{r}, t)$, parallel spin $\delta \mathbf{S}_{\|}(\mathbf{r}, t)$ and vertical spin $\delta S_{z}(\mathbf{r}, t)$ for a given oscillation mode and a fixed time (Fig. 3), where we define the spin density as $\mathbf{S}(\mathbf{r})=\sum_{i=1}^{N}\left\langle\chi_{i}\left|\vec{\sigma} \delta\left(\mathbf{r}-\mathbf{r}_{i}\right)\right| \chi_{i}\right\rangle$. This local-signal analysis has been performed using the method of Ref. [12]. Quite remarkably, the oscillation of $\delta S_{z}(\mathbf{r}, t)$ is always in a perpendicular direction to that of the charge density, which is explained in the analytical model as a coherent quasi-up and quasi-down excitation due to the SO term. On the contrary, the parallel spin patterns depend on whether the mode has quasi-spin flip character or not. For the split Kohn modes (peaks a and b) $\delta \mathbf{S}_{\|}(\mathbf{r}, t)$ is localized in the two regions perpendicular to the charge oscillation axis. However, the quasi-spin flip peak (c) shows no angular dependence of the pattern. It can be shown that this behaviour is in complete agreement with the prediction of the analytical model using the spinors of Eq. (5).

We consider next how the features discussed above are modified by the addition of the Coulomb interaction

$$
\mathcal{H}_{C}=\sum_{i<j}^{N} \frac{e^{2}}{\kappa\left|\mathbf{r}_{i}-\mathbf{r}_{j}\right|},
$$

where $\kappa$ is the semiconductor dielectric constant $(\kappa=$ 12.4 for GaAs). We shall estimate interaction effects using a selfconsistent mean-field for the Hartree electrostatic potential

$$
V_{H}(\mathbf{r})=\frac{e^{2}}{\kappa} \int d \mathbf{r}^{\prime} \frac{\rho\left(\mathbf{r}^{\prime}\right)}{\left|\mathbf{r}^{\prime}-\mathbf{r}\right|},
$$

and the LSDA for electronic exchange and correlation. Within the present spinor formalism the dots have a vectorial spin magnetization $\mathbf{S}(\mathbf{r})$ and, therefore, a general exchange-correlation functional of the type $E_{X C}[\rho, \mathbf{S}]$ is necessary. For this we have resorted to the non-collinearspin formulation of LSDA theory, described for instance in Ref. 81, and employed the Monte-Carlo results of Ref. [13] for the bulk electron gas.

The modifications stemming from the Coulomb interaction are twofold; first, the electron-hole transitions move in energy due to the change in the effective mean field and; second, new collective peaks may appear as a result of coherent electron-hole excitations. Figure 4 shows the interacting results for $N=6$ with $z_{0}=50$ and $35 \AA$. Note that the splitting of the Kohn-like modes 
disappears for the larger width and, at the same time, a collective peak begins to appear at an energy below the electron-hole states. These results clearly show the formation of the collective plasmon and magnon states lying above and below the electron-hole transitions, respectively. We stress that in the absence of SO coupling the magnon state does not contribute to the FIR absorption. The lower left panel of Fig. 4 indicates that a great enhancement of the magnon contribution is obtained by reducing the dot width, which is accompanied by an important Landau damping of the plasmon state into a bundle of peaks. The Coulomb interaction thus suppresses the splitting of the Kohn-like modes although it introduces the mechanism of Landau damping that ultimately, and rather abruptly with $z_{0}$, dominates for decreasing widths.

The right panels of Fig. 4 show the horizontal spin spectra induced by the dipole shift. These results permit to ascertain the spin character of the different modes and, by comparison with the left panels, quantify their relevance to the FIR absorption. The relative spin or density character of each state is also seen from the amplitudes $\mathcal{A}$ of density and spin oscillation. For instance, when $z_{0}=50 \AA$ we find $\mathcal{A}\left[\delta S_{z}(\mathbf{r}, t)\right] \approx 60 \mathcal{A}[\delta \rho(\mathbf{r}, t)]$ for the magnon and $\mathcal{A}\left[\delta S_{z}(\mathbf{r}, t)\right] \approx 0.4 \mathcal{A}[\delta \rho(\mathbf{r}, t)]$ for the plasmon, which shows the dominant spin and density type for magnon and plasmon states, respectively.

The characterization of the interacting peaks is facilitated by the comparison of the spatial patterns with the corresponding ones in the non-interacting model. Figure 5 displays the density and spin patterns for two of the peaks of the $z_{0}=35 \AA$ spectrum (lower left panel of Fig. 4). As in the non-interacting model, the oscillation of $\delta S_{z}(\mathbf{r}, t)$ is orthogonal to that of $\delta \rho(\mathbf{r}, t)$ and the horizontal spin patterns show a priviledged spin direction, i.e., they have a net induced spin. Note in particular that peak b of Fig. 4 has no angular dependence in the horizontal spin pattern while for peak a there are two regions with enhanced $\delta \mathbf{S}_{\|}(\mathbf{r}, t)$. We have checked that these features are not changed when the polarization of the mode, given by the direction of the density oscillation, is varied. We stress that the mechanism discussed here would permit a control of the horizontal spin oscillation by tuning the frequency of the applied electric field. This effect of spin-to-charge conversion is a major requisite for hybrid spintronic devices [2].

Finally, Fig. 6 presents the spectra with Coulomb interaction obtained for $N=10$, an open-shell system in the analytical model. At $z_{0}=50 \AA$ the open-shell character of this dot is clearly maintained, with low-energy electron hole transitions and an associated collective mode at $\omega \approx 0.7 \mathrm{meV}$ similar to the quasi-spin flip excitations of Fig. 1. For narrower dots, however, the energy of quasispin flip transitions increases and as a result this mode merges with the magnon and other electron-hole states. Contrary to the quasi-spin flip state, both magnon and plasmon mean energies are not dependent on the Dresselhaus coupling.

To summarize, the role of the SO coupling in the FIR absorption of GaAs dots has been studied. In an analytical model without interaction the relevant Dresselhaus contribution induces characteristic splittings and quasi-spin flip modes. The Coulomb interaction favors the formation of collective plasmon and magnon states and it suppresses the splittings of the analytical model. Nevertheless, below a certain dot width Landau damping dominates the spectrum. Each mode has characteristic patterns of induced charge, horizontal spin and vertical spin, as well as a ratio of oscillating density and spin amplitudes. Therefore, by tuning the frequency of the applied electric field one can effectively control the dynamical spin properties and the relative intensities of the charge and spin oscillation.

This work was supported by Grant No. PB98-0124 from DGESeIC, Spain.

[1] S. Datta and B. Das, Appl. Phys. Lett. 56, 665 (1990).

[2] S. A. Wolf, D. D. Awschalom, R. A. Buhrman, J. M. Daughton, S. von Molnár, M. L. Roukes, A. Y. Chtchelkanova, D. M. Treger, Science 294, 1488 (2001).

[3] J. A. Folk, S. R. Patel, K. M. Birnbaum, C. M. Marcus, C. I. Duruöz, J. S. Harris, Phys. Rev. Lett. 86, 2102 (2001).

[4] B. I. Halperin, A. Stern, Y. Oreg, J. N. H. J. Cremers, J. A. Folk, C. M. Marcus, Phys. Rev. Lett. 86, 2106 (2001).

[5] I. L. Aleiner and V. I. Fal'ko, Phys. Rev. Lett. 87, 256801 (2001).

[6] O. Voskoboynikov, C. P. Lee, O. Tretyak, Phys. Rev. B 63, 165306 (2001).

[7] W. Knap, C. Skierbiszewski, A. Zduniak, E. LitwinStaszewska, D. Bertho, F. Kobbi, J. L. Robert, G. E. Pikus, F. G. Pikus, S. V. Iordanskii, V. Mosser, K. Zekentes, Yu. B. Lyanda-Geller, Phys. Rev. B 53, 3912 (1996).

[8] O. Heinonen, J. M. Kinaret, M. D. Johnson, Phys. Rev. B 59, 8073 (1999).

[9] T. Darnhofer and U. Rössler, Phys. Rev. B 47, 16020 (1993).

[10] L. Jacak, J. Krasnyj, A. Wójs, Physica B 229, 279 (1997).

[11] A. Puente, Ll. Serra, Phys. Rev. Lett. 83, 3266 (1999).

[12] M. Valín-Rodríguez, A. Puente, and Ll. Serra, Phys. Rev. B 64, 205307 (2001).

[13] B. Tanatar and D. M. Ceperley, Phys. Rev. B 39, 5005 (1989). 


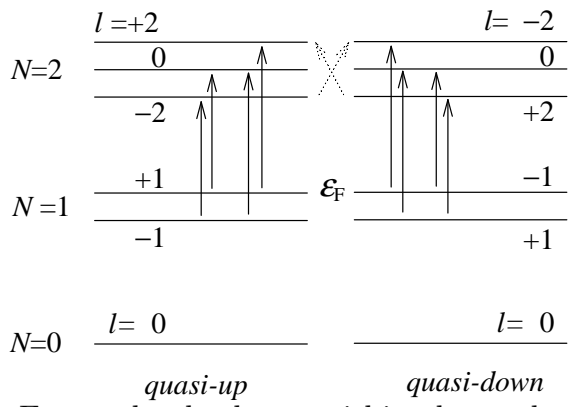

FIG. 1. Energy level scheme within the analytical model for $\mathcal{H}_{0}+\mathcal{H}_{D}$. The Fermi level for $N=6$ is indicated by $\varepsilon_{F}$ and the vertical arrows correspond to the allowed transitions having $\Delta \ell= \pm 1$ within each quasi-spin subset. The dotted lines show quasi-spin flip $\Delta \ell=0$ transitions within the $N=2$ major shell, discussed in the text for $N=10$.
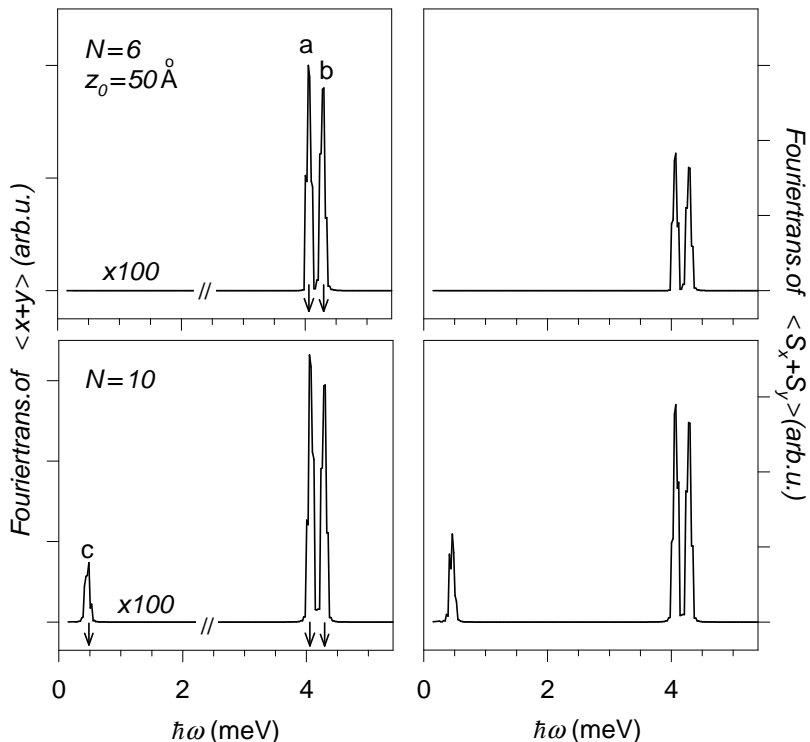

FIG. 2. FIR absorption (left panels) obtained from the Fourier transform of the real time dipole oscillation with $\mathcal{H}_{\text {full }}$ for a dot width of $z_{0}=50 \AA$. Right panels display the corresponding frequency transform of the horizontal spin signal. The arrows indicate the energies of the split Kohn modes (at $\approx 4 \mathrm{meV}$ ) and of the quasi-spin flip modes (at $\approx 0.5 \mathrm{meV}$ ) using the analytical model for this $z_{0}$.

FIG. 3. Spatial patterns of oscillating density $\delta \rho(\mathbf{r}, t)$, horizontal spin $\delta \mathbf{S}_{\|}(\mathbf{r}, t)$ and vertical spin $\delta S_{z}(\mathbf{r}, t)$ at a fixed time for the a and $\mathrm{c}$ dipole modes of Fig. 1. The gray color scale of left and right panels indicates the signal magnitude while the corresponding sign is superimposed in white.
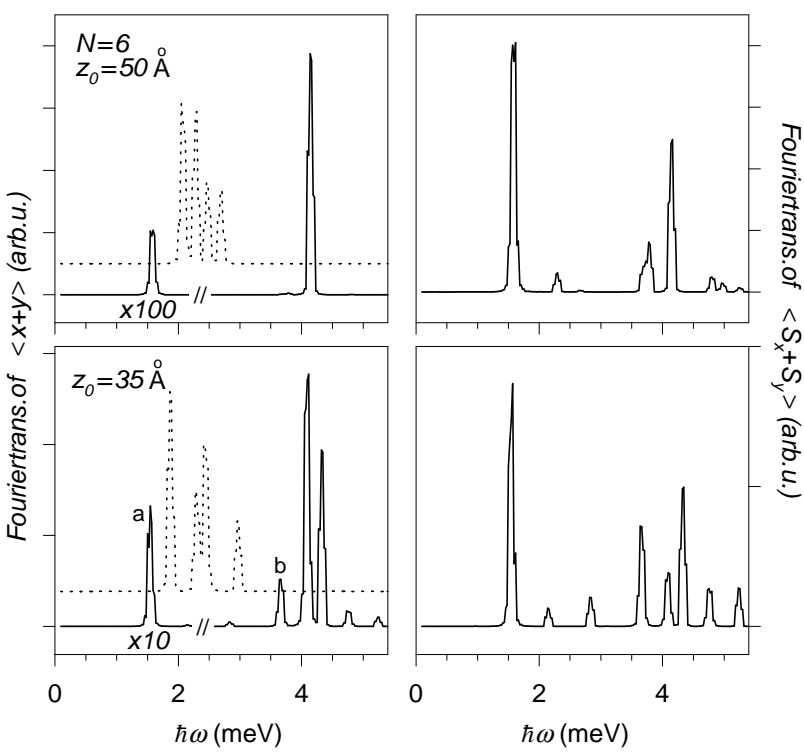

FIG. 4. Same as Fig. 2 including Coulomb interactions The dashed line in the left panels displays the electron-hole transitions in the selfconsistent mean-field.

FIG. 5. Same as Fig. 3 including Coulomb interaction. Upper and lower row correspond to the peaks labeled a and b in Fig. 4, respectively.

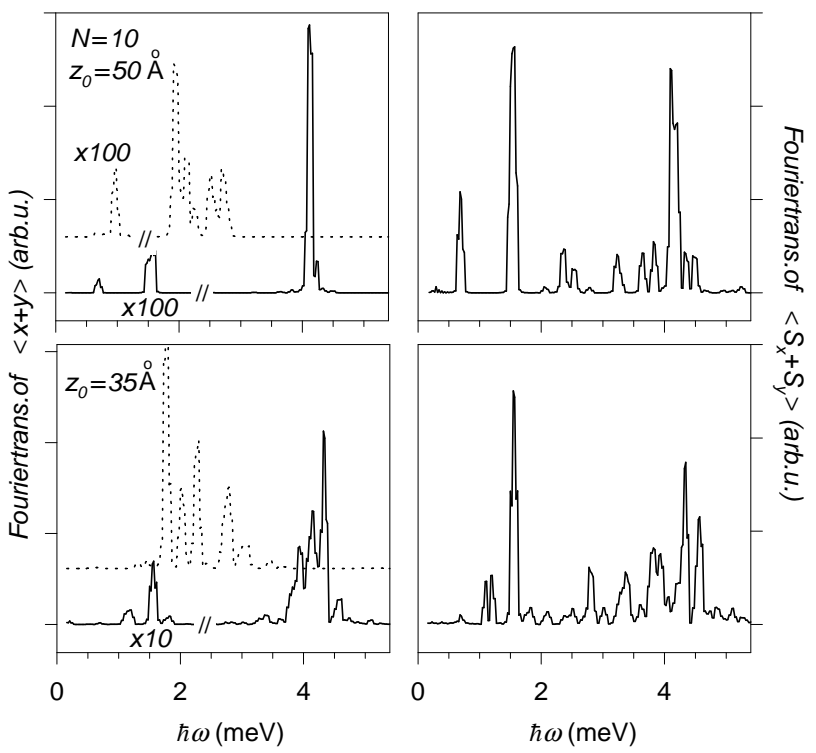

FIG. 6. Same as Fig. 4 for $N=10$. 
This figure "fig3.jpg" is available in "jpg" format from: http://arxiv.org/ps/cond-mat/0204256v1 
This figure "fig5.jpg" is available in "jpg" format from: http://arxiv.org/ps/cond-mat/0204256v1 